\title{
DUST AND THE GAS PHASE COMPOSITION OF DENSE CLOUDS
}

\author{
C. M. WALMSLEY \\ Max Planck Institut für Radioastronomie
}

\begin{abstract}
A review is given of our current knowledge of the gas phase abundance of interstellar molecules. A comparison is made between cold dust clouds, where presumably gas phase processes dominate the chemistry, and regions of high mass star formation such as Orion where both shocks and evaporation of dust grain mantles may play a role. In dust clouds, carbon rich molecules often show enhanced abundances, whereas in hot turbulent star formation regions, saturated species such as methanol and ammonia are often favored. In both types of source, there is evidence for abundance gradients and inhomogeneities. A probable cause is that the chemistry is far from a steady state situation. A useful diagnostic in such cases is the relative abundance of deuterated species and our current knowledge concerning deuterium fractionation is discussed. Recent results show that the observed degree of deuterium enhancement in species such as water, methanol, and ammonia in the Orion-KL region is much too great to be explained on the basis of normal gas phase chemistry. It is argued that a plausible explanation of the observations is that one is observing material which has recently been evaporated from dust grain mantles. One important consequence of this hypothesis is that molecular line observations may yield important information on the composition of the solid phase.
\end{abstract}

\section{INTRODUCTION}

In this brief review of the current state of our knowledge concerning gas phase molecular abundances and their interpretation, I can only give a sketchy account of the basic principles of interstellar chemistry. I will try to focus upon a few issues which are of interest for the "dust community." However, there are many good reviews of interstellar chemistry and related topics. The basic principles both of grain surface and gas phase chemistry are well summarized in the book by Duley and Williams (1984). The reviews by Watson $(1976,1978)$ give another useful account of the basic physical principles involved in interstellar molecule formation while Millar (1988) summarizes recent progress in modelling. On the observational side, the article by Irvine, Goldsmith, and Hjalmarson (1987) gives a detailed discussion of the difficulties and errors in column-density determination. They also give a very useful tabulation of the current best estimates of the molecular abundances in sources such as Orion-KL and the Taurus Molecular Cloud 1 (hereafter known as TMC1). A comparison of observation and theory is given by Walmsley (1985, 1986) where emphasis is laid on the successes and failures of gas phase chemistry models 
The present article will concentrate on what one has learned or can hope to learn about the formation of ice mantles from observations of interstellar molecules. However, I will also examine to what extent current gas phase models are capable of explaining observations. I therefore discuss in Section 2 of this article the current observational situation for two well studied sources (Orion and TMC1) and in Section 3, I discuss some of the attempts to model these sources. In Section 4, I discuss the hypothesis that in sources such as Orion-KL, much of the gas which we observe in the so-called "hot core" is freshly evaporated dust mantle material. This is presently unproven but if correct, it has considerable consequences. One of these is that we have the possibility to establish the compositions of grain mantles on the basis of molecular line observations. Another is that we can get some information on the time which has elapsed since the switch-on of young stars in sources such as Orion-KL. Finally, in Section 5, I briefly discuss the critical problem of methanol which the infrared data suggest may be a major constituent of mantles.

\section{OBSERVATIONS OF ORION-KL AND TMC1}

The contrasting sources Orion and TMC1 have been used as standard objects by theoreticians attempting to model interstellar chemistry. TMC1 first attracted interest because it was discovered that long chain carbon-rich compounds such as the cyanopolyynes $\mathrm{HC}_{2 n+1} N$ could readily be observed there. TMC1 has additionally the characteristic that the gas within it is very quiescent with line-widths of order 0.3-0.4 $\mathrm{km} \mathrm{s}^{-1}$. When mapped in a high density tracer such as $\mathrm{NH}_{3}$, one sees that the source has a highly elongated structure (see Olano et al., 1988) and it has been speculated that it may be a disk seen edge on. Because of the quiescence and low temperature $(10 \mathrm{~K})$, it has generally been assumed that only gas phase reactions can be of importance for molecule formation in this source. Grains influence the chemistry only in that they protect the molecules from the external ultraviolet radiation field and in that molecules can potentially freeze out on their surfaces.

The situation is quite different in a hot turbulent source such as Orion-KL. Here, the temperature is sufficiently high ( $\gtrsim 100 \mathrm{~K}$ ) that one can expect evaporation of dust mantles. There is moreover abundant evidence of shocked gas in, and around, Orion-KL. This is of fundamental importance for the chemistry. At temperatures above $1000 \mathrm{~K}$, a variety of reactions which either require surmounting an activation energy barrier or are endothermic become possible. Above $1000 \mathrm{~K}$ also, emission in the vibrationally excited lines of molecular hydrogen is an important cooling process for the gas and indeed these transitions are easily observed in the region surrounding Orion-KL. One concludes that the outflow from the young star, OriIRC 2, is interacting with surrounding gas. One can expect that the observed molecular abundances should be strongly affected by this shock processing. The timescales for most gas-phase chemical processes are long and hence the medium will take a long time to relax back to the steady-state composition appropriate to it's density and temperature. The same applies to the consequences of evaporating dust grain mantles and it is this "long memory" which complicates the interpretation of the observed abundances in Orion-KL.

Given that Orion and TMC1 have highly contrasting properties, it is interesting to compare the observed abundances in the two sources. This is done in Figure 1 which is based mainly upon the compilation of Irvine, Goldsmith, and Hjalmarson (1987). With some exceptions, one sees that many molecules have similar abun- 
dances in the two sources. However, it is noticeable that methanol $\left(\mathrm{CH}_{3} \mathrm{OH}\right)$ is much more abundant in Orion and that all carbon-rich species are more abundant in TMC1. There is also a tendency for radicals such as $C N$ and $C_{2} H$ to be more abundant in cold clouds whereas more saturated (hydrogenated) species are seen in Orion. Hence one might conclude directly from the observations that gas phase chemistry alone should be capable of accounting for the long chain carbon compounds while other processes are operating in regions such as Orion.

Before discussing models however, I should stress that comparison of theory and observation is complicated by the extreme inhomogeneity of sources such as TMC1 and, in particular, Orion. The structure of TMC1 and abundance variations within the source are discussed by Olano et al. (1988) and by Walmsley and Wilson (1984). Orion is even more complex (see the review of Welch, 1988). The young massive pre-main-sequence star IRC 2 is losing mass rapidly in a bipolar flow to the south-east and north-west. The gas observed associated with this flow is normally referred to as plateau gas while a very high density molecular clump (size $0.03 \mathrm{pc}$ ) close to IRC 2 and heated by it is called the hot core. Hot core temperatures are thought to range between $150 \mathrm{~K}$ and $250 \mathrm{~K}$ and the density must be at least $10^{7}$ $\mathrm{cm}^{-3}$. Rather lower temperatures $(70-100 \mathrm{~K})$ pertain in the more extended region known as the Orion ridge. It seems possible that this ridge consists essentially of a series of clumps similar to but with lower densities and temperatures than the hot core. A dense clump a few arc seconds (rich in methanol, see Wilson et al., 1988) to the south of the hot core is known as the compact ridge. Interferometer maps which are gradually becoming available (see e.g. Mundy et al., 1986) are giving a more detailed picture of the region and maps in different species (see e.g. Welch, 1988; Plambeck and Wright, 1988) show a great variety of structures. Some of this variety is due to excitation effects but there are real variations in abundance. These must be borne in mind when comparing theoretical models with observed single dish column densities .

\section{MODEL CALCULATIONS AND THEIR ASSUMPTIONS}

In this section, I summarize the results of some recent model calculations of the evolution of gas phase molecular abundances. It is useful first however to note that one of the problems in interpreting the observations is that there are at least three competing timescales involved. In the first place, the gas phase chemistry in dense clouds is driven by cosmic ray ionization which gives rise to the molecular ions which are characteristic of that chemistry. The best known example is $\mathrm{HCO}^{+}$. A characteristic timescale for the gas-phase chemistry is the time required to convert free gas-phase atomic carbon into $C O$ which is typically a few times $10^{6}$ years. (However many less abundant species evolve faster). Gas-phase processes compete with freeze-out onto interstellar grain surfaces which should occur (see Duley and Williams, 1984) on a timescale of $3 \times 10^{9} / n$ years where $n$ is the hydrogen density in $\mathrm{cm}^{-3}$. In dense cloud cores therefore with densities $n \gtrsim 10^{4} \mathrm{~cm}^{-3}$, one can expect that grain mantle depletion will occur on a timescale faster than that required for the gas-phase chemistry to reach a steady-state. A consequence is that the composition of deposited mantle material depends not only on the molecular cloud density and temperature but also on the initial conditions and history of the region.

An added complication is that dynamical timescales may also be short. It seems reasonable to suppose that a minimum lifetime for a dense clump or core is given 


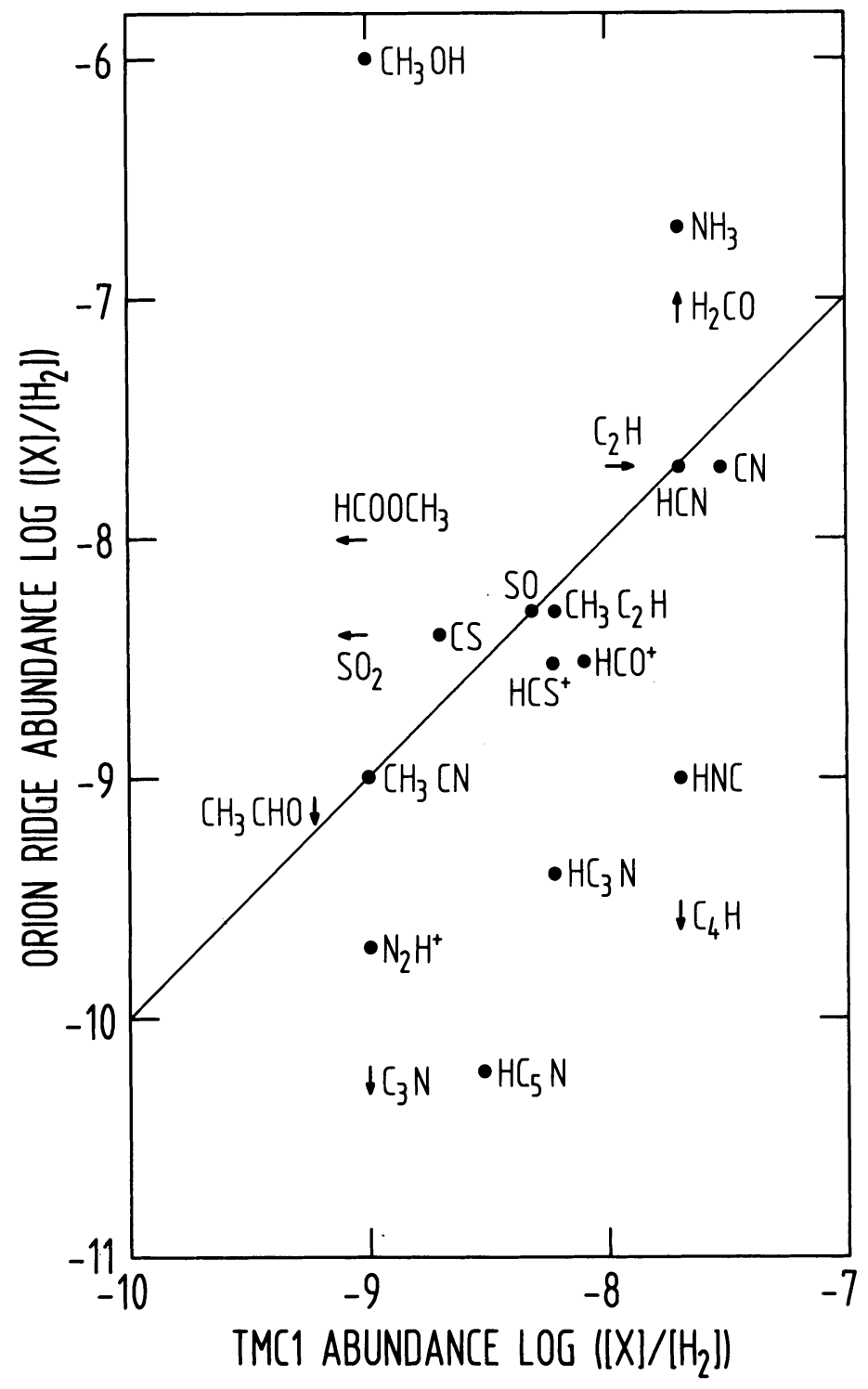

Fig. 1. The observed Orion ridge relative abundances $(\mathrm{T} \sim 100 \mathrm{~K})$ are plotted against observed abundances in the cold dark core TMC1. The data are mainly taken from Irvine et al. 1987. The full-line denotes equal abundance in the two sources. 
by the free-fall timescale or $2 \times 10^{7} / n^{0.5}$ years. Most such regions appear to be virialized (see e.g. Myers, 1983) and hence this is not greatly different from the sound crossing time. It is an interesting coincidence that this dynamical timescale is of the same order as the freeze-out timescale for typical clump densities. At higher densities, freeze-out should occur more rapidly than dynamical evolution. One conclusion which one might draw is that the age of such clumps is unlikely to be much greater than a free-fall timescale since the evidence indicates that only a moderate amount of depletion has occurred in most cores. (There are no known examples of clumps observable in dust extinction but without molecular counterparts.) Moreover, Beichman et al., 1986 find that $~ 50$ percent of the clumps studied by them have embedded IRAS sources. This implies that most clumps eventually give rise to low-mass stars and that the free-fall time is indeed a good rough measure of the lifetime of a clump. From the point of view of molecular abundance studies, this has the unfortunate consequence that, not only are steady-state solutions of the gas-phase chemistry irrelevant, but also in principle, one should consider both clump dynamics and mantle deposition when solving for the evolution in time of molecular abundances.

Such a procedure is of course not normally followed. Most theoretical studies have concentrated on the gas phase chemistry and have neglected freeze-out and dynamics. It is usual to solve the "pseudo time-dependent" problem which implies solving the equations governing the time evolution of molecular abundances assuming fixed density, temperature, and free (i.e. gas phase) elemental abundances. There are many solutions of this type and I refer to recent articles by Herbst and Leung (1988), by Langer and Graedel (1988), and references therein for a more detailed discussion. The review by Millar (1988) discusses some of the key reactions and assumptions.

One of the main aims of such studies has been to explain the surfeit of longchain carbon compounds (see Fig. 1) observed in dense condensations such as TMC1. Models of this type have become increasingly ambitious. The model of Herbst and Leung (1988) for instance contains 2548 reactions and considers 273 different species. Molecules as complex as $\mathrm{HC}_{9} \mathrm{~N}$ are considered. One finds, interestingly enough, that starting with atoms, one can produce complex molecules in roughly the observed abundances but only at "early times," well before steady-state is realized. Over timescales much longer than 1-2 x $10^{5}$ years, the available gas phase carbon becomes converted into $C O$ and the abundances of complex hydrocarbons rapidly drop. The cyanopolyyne abundance is thus a measure of age and one might speculate that TMC1, in comparison to other dark cores, is particularly young. In any case, the results are consistent with the hypothesis that dark cores exist for, at most, a few free-fall times. Fig. 2 shows a comparison of the Herbst-Leung early times result with observations. One sees that most species are reasonably (order of magnitude) accounted for.

When modelling regions such as Orion-KL, it seems more realistic to consider mantle deposition and evaporation. In this case, grain surface reactions presumably become important. The available studies (Tielens and Hagen, 1982; d'Hendecourt et al., 1985) show in general that atomic hydrogen addition reactions leading to saturated species are important. Heavier species will be less mobile on the grain surface and are likely to be unreactive. Thus if atomic hydrogen is abundant in the gas phase, one is likely to produce ices of water, methane, ammonia etc. in the grain mantle. On the other hand, at the densities of interest, atomic hydrogen may 


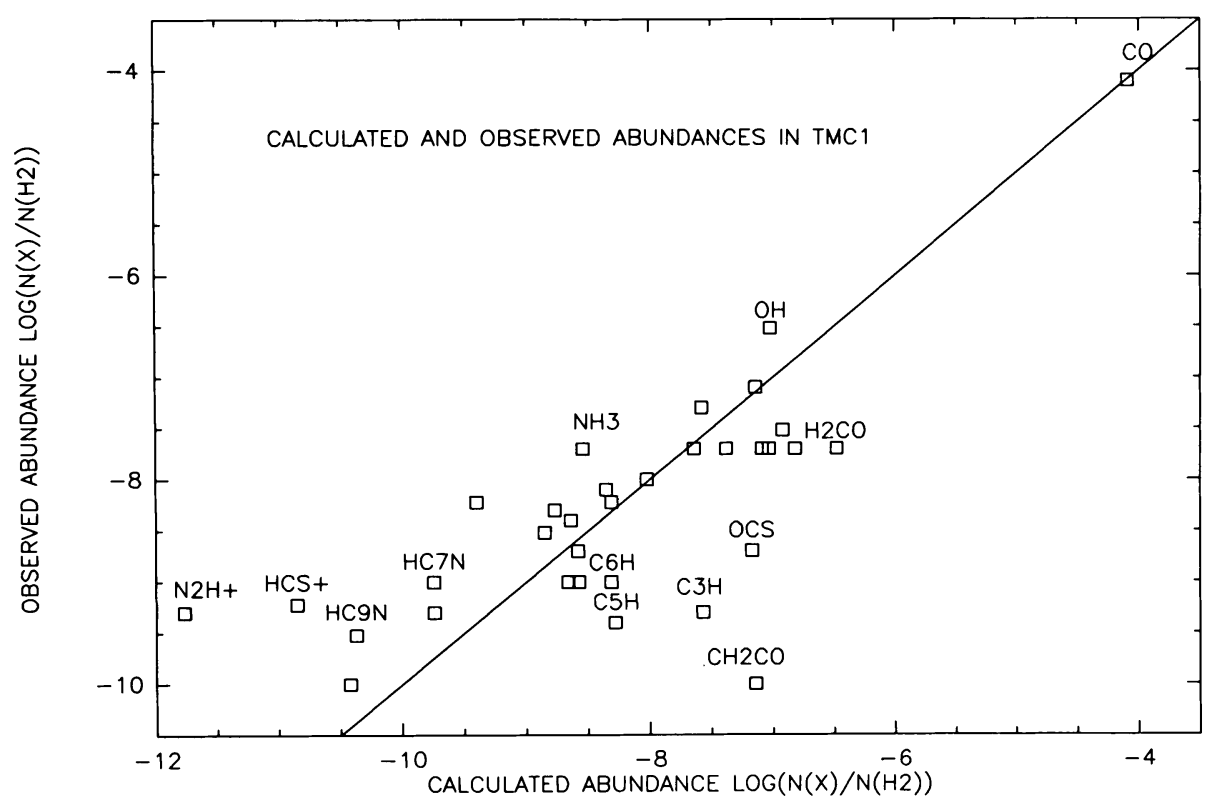

Fig. 2. Comparison of the Herbst and Leung, 1988 model predictions for TMC1 with observed abundances. The early times model has been used.

not be very abundant and the dust mantle composition may reflect more accurately the gas phase molecular distribution.

An ambitious recent model of the Orion hot core chemistry is that of Brown et al., 1988. They have followed both gas phase and surface chemistry in a clump which collapses starting from an initial density of $3000 \mathrm{~cm}^{-3}$. The collapse is halted after all condensible species have been accreted and then a young star is "switched on" which heats it's surroundings to temperatures of 200K. This removes the grain mantle and one thus has new initial conditions for the gas phase chemistry. The computations follow the development at a density of $10^{7} \mathrm{~cm}^{-3}$ for the next $10^{4}$ years. The results (see Fig. 3) should probably be considered as illustrative. They suggest that, even at high densities, a mantle composition consisting essentially of water, methane, and ammonia ices is plausible. Comparison with observations suggest in fact that the models are producing too much water and ammonia. Recent observations of $\mathrm{H}_{2}^{18} \mathrm{O}$ by Jacq et al. (1988) suggest that the abundance of water relative to hydrogen in hot core regions is of order $10^{-5}$ and thus smaller than that of $\mathrm{CO}$. This is in contrast with the models suggesting that $\mathrm{H}_{2} \mathrm{O}$ ice is not the main form of oxygen in grain mantles. 
ORION HOT CORE MODEL AND OBSERVED ABUNDANCES

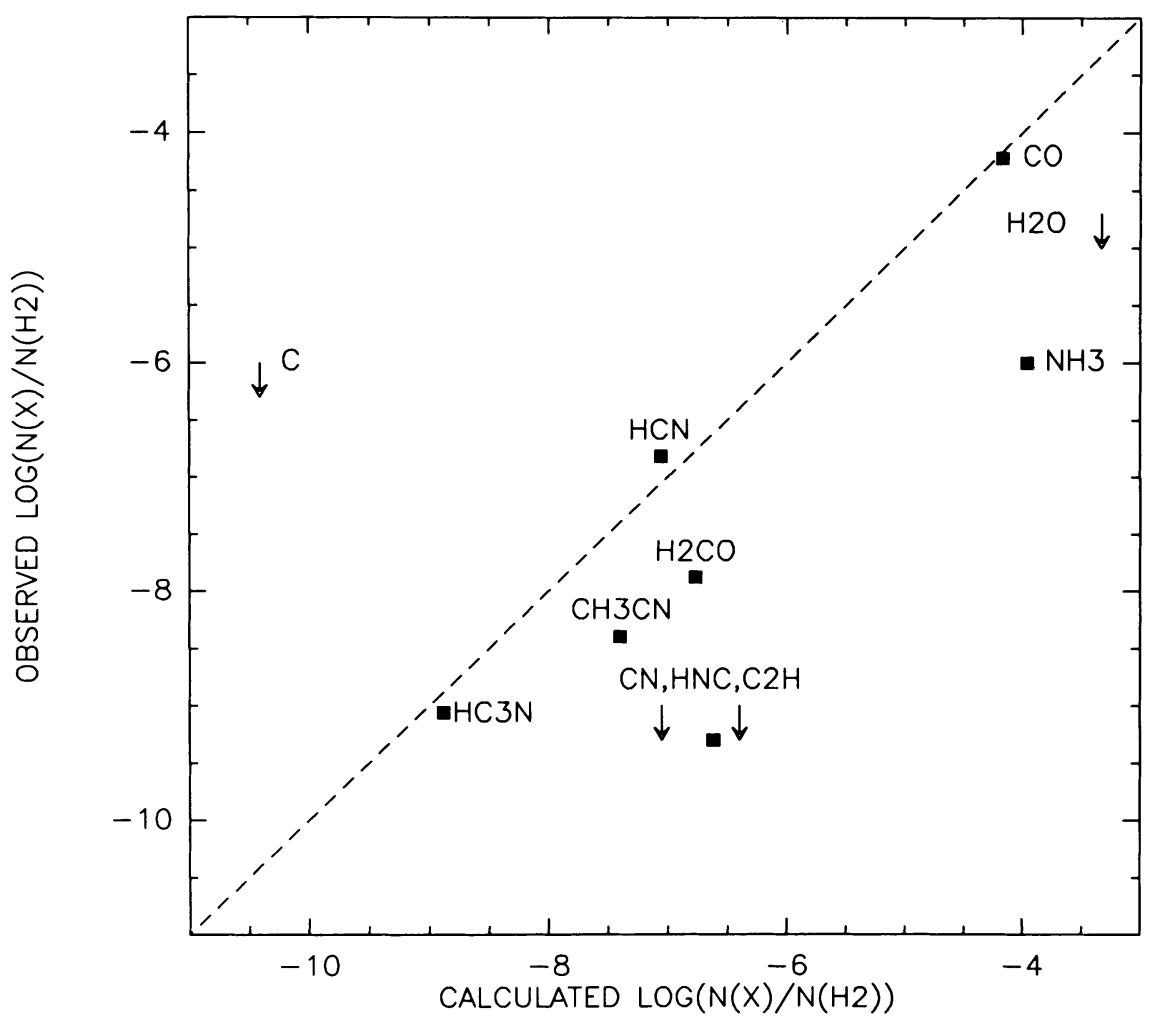

Fig. 3. Comparison of observations and model predictions for the Orion hot core. The model results are taken from Brown et al., 1988 and the data are as cited by them (For $\mathrm{H}_{2} \mathrm{O}$, see Jacq et al., 1988). The dashed-line corresponds to agreement between theory and observation.

\section{DEUTERIUM FRACTIONATION IN HOT CORES}

In regions such as the Orion hot core, a variety of processes are clearly responsible for the observed abundance distribution. The article by Blake et al., 1987 summarizes many of these. It is clear that both shocks and grain mantle evaporation play a role and one must attempt to estimate the relative importance of these two mechanisms. A precise analysis will probably only be possible once interferometer maps of the column density distribution in Orion-KL become available. However, the available data already allow some qualitative conclusions to be drawn (see Welch, 1988). Also, one can devise some observational tests which allow a rough estimate of the extent to which various processes dominate. One such test is the amount of decterium fractionation which (see reviews by Wootten (1986) and Walmsley (1985)) is known to be large in molecular clouds. Another is the $[H C N] /[H N C]$ abundance ratio which varies rapidly in the region around Orion-KL (see Goldsmith et al., 1986). 
Abundance ratios of this kind are relatively easy to determine observationally and can be expected to show different characteristics in post-shock gas and in dust mantle material. Here, I will confine myself to a brief discussion of the conclusions one can draw from the observed deuterium enrichment in hot $(T \gtrsim 100 \mathrm{~K})$ molecular cores.

In cold clouds where deuterium enrichment is thought to be understood, the key reaction is the exchange:

$$
\mathrm{H}_{3}^{+}+\mathrm{HD} \rightleftharpoons \mathrm{H}_{2} \mathrm{D}^{+}+\mathrm{H}_{2}
$$

This reaction is exothermic by an amount $\Delta \mathrm{E} \sim 200 \mathrm{~K}$ and hence, under interstellar conditions, the forward reaction dominates. One consequently obtains an enrichment in the ratio $\left[\mathrm{H}_{2} \mathrm{D}^{+}\right] /\left[\mathrm{H}_{3}^{+}\right]$relative to the interstellar $\mathrm{D} / \mathrm{H}$ ratio of roughly three orders of magnitude (See Lepp and Dalgarno, 1984; Herbst, 1982; Brown and Rice, 1986; and Walmsley, 1986). This can be passed on to other species by reactions of the type:

$$
\mathrm{H}_{2} \mathrm{D}^{+}+\mathrm{CO} \rightarrow \mathrm{DCO}^{+}+\mathrm{H}_{2}
$$

$\mathrm{DCO}^{+}$is a known interstellar molecule and its abundance in cold clouds such as TMC1 can be reasonably well explained in terms of reactions 1 and 2 . This is also the case for other deuterated molecules (See Millar et al., 1988) and, in fact, the only species which seems to be deviant is $C_{3} H D$. This molecule appears to have a ratio $\left[\mathrm{C}_{3} \mathrm{HD}\right] /\left[\mathrm{C}_{3} \mathrm{H}_{2}\right]$ as high as 0.1 in some cold cores (Bell et al., 1988) which is difficult to account for with current theories.

At higher temperatures, other reactions with higher exothermicity than (1) become important in the deuterated chemistry. The study of Millar et al., 1988 shows that a ratio $[\mathrm{HDO}] /\left[\mathrm{H}_{2} \mathrm{O}\right]=0.001$ can plausibly occur at a temperature of $70 \mathrm{~K}$ in a purely gas-phase chemistry. This is also true for several other species but not for $\left[\mathrm{NH}_{2} \mathrm{D}\right] /\left[\mathrm{NH}_{3}\right]$ which is at most $4 \times 10^{-4}$ under these circumstances. Figure 4 gives a comparison of calculated and observed deuterated ratios for the Orion ridge (Some of the observed values may refer to the hot core). One sees that the observed deuterated ratios are higher than the model predictions but the general species to species variation is reproduced. This could mean that the ridge molecules were formed in a region whose temperature was lower than $70 \mathrm{~K}$.

However, the evidence suggests that some at least of the deuterated molecules are abundant in the hot core region of Orion where the temperature is thought to exceed 100K. HDO is one example (See Plambeck et al., 1987; Henkel et al., 1987) and $\mathrm{NH}_{2} \mathrm{D}$ is another (Walmsley et al., 1987). Both the excitation of these species and their spatial distributions suggest that they exist in regions with temperatures above $100 \mathrm{~K}$ and densities above $10^{6} \mathrm{~cm}^{-3}$. This is very hard to explain in any purely gas phase scheme with temperatures corresponding to the observed values. It is also expected that post-shock gas will not show deuterium fractionation (Moore et al., 1986). One is therefore led to the possibility that the gas in Orion-KL has only recently been heated to the observed temperatures and that the chemistry has not had time to adjust to the changed situation. The observed deuterated ratios are representative of an epoch prior to the switch-on of IRC 2 when the temperature was low. Given that saturated species such as ammonia are known to be in excess in the Orion hot core, it is also natural to attempt to explain the chemistry in terms of evaporation of dust grain mantles. In this case, the observed 


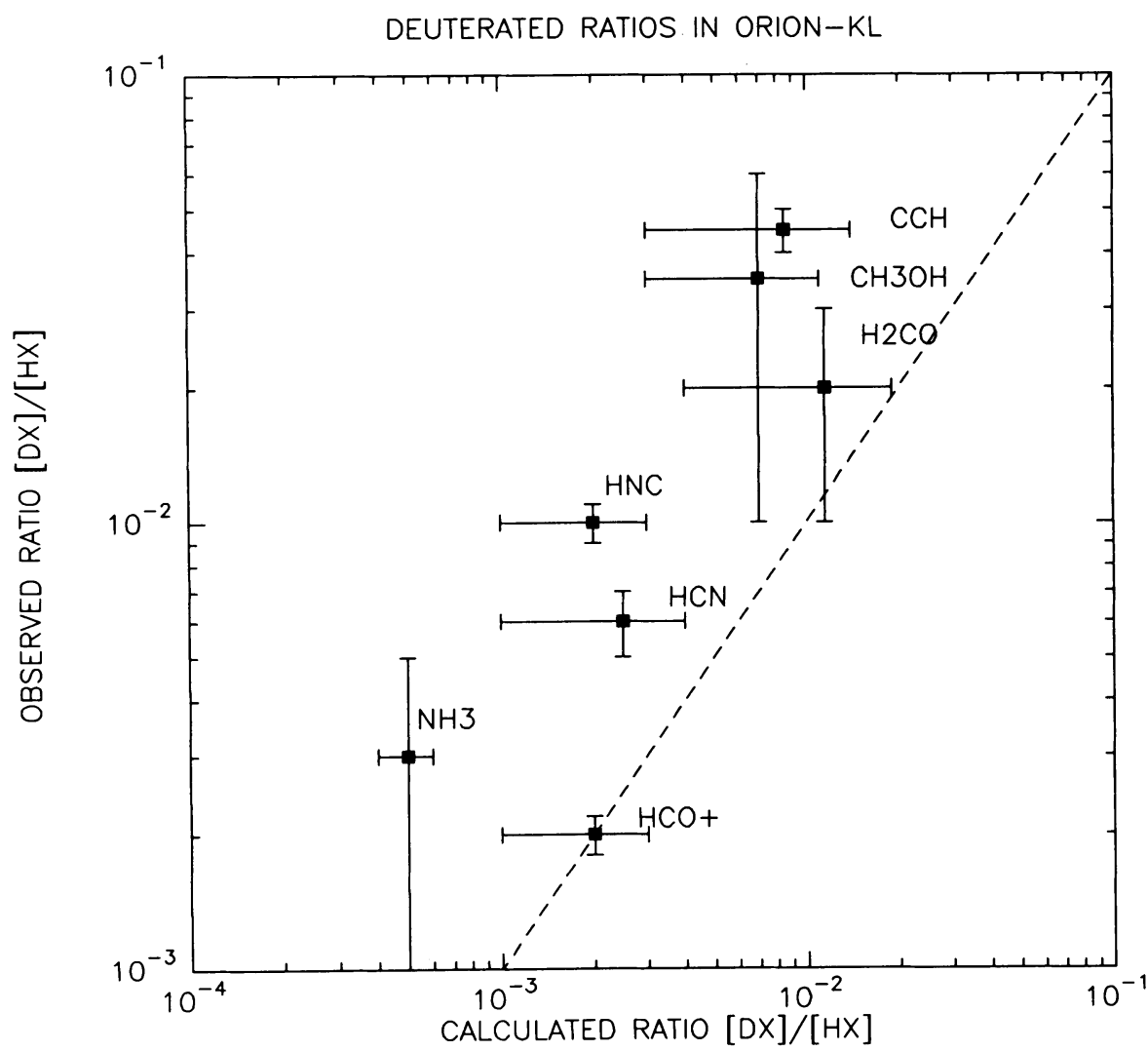

Fig. 4. A comparison of observed and calculated deuterated ratios for the Orion ridge. The theoretical predictions are taken from the Millar et al., 1988 early times models for a temperature of 70K. Agreement between observation and theory occurs for points close to the dashed-line

deuterium fractionation is influenced both by the gas phase chemistry prior to mantle deposition and by reactions on grain surfaces.

The degree of deuterium fractionation in such a scenario will also be influenced by reactions of $D$ atoms on grain surfaces (Tielens, 1983). The gas phase processes discussed earlier will not only enhance the abundance of deuterium containing molecules but will also cause enrichment of atomic deuterium relative to atomic hydrogen. This is in particular due to processes of the type:

$$
\mathrm{DCO}^{+}+e \rightarrow \mathrm{D}+\mathrm{CO}
$$

The enrichment of $\mathrm{DCO}^{+}$by reactions (1) and (2) thus translates into an enhancement of $[D \mathrm{I}] /[H \mathrm{I}]$ and there are therefore relatively more $D$ atoms available than $H$ atoms for surface chemistry. On the grain surface in the models of Tielens (1983), $D$ addition can lead to large abundances of species such as $\mathrm{NH}_{2} \mathrm{D}$ and $\mathrm{HDCO}$. 
Indeed, the prediction is that $\mathrm{NH}_{2} \mathrm{D}$ could be more abundant than $\mathrm{NH}_{3}$ in the mantle material. However this result is dependent on the accuracy of the treatment of the $D$ atom chemistry which is somewhat uncertain.

However, deuterium fractionation in hot cores is not by itself a proof that grain mantle evaporation is occurring. It is merely an indication of a sudden rise in temperature. The test for any grain mantle evaporation theory is whether one can explain both the deuterated molecules and the abundances of species such as ammonia which (See Fig. 1) are anomalously abundant in hot regions. As discussed in the previous section, models such as that of Brown et al., 1988 overproduce $\mathrm{NH}_{3}$. Another important test is to reproduce the observed ratio of $[H C N] /[H N C]$ which is very high in the immediate neighborhood of Orion-KL (Goldsmith et al., 1986). Here, it is of importance to find out whether $H N C$ can isomerize to $H C N$ on grain surfaces (e.g. by reaction with $H$ atoms)

\section{EXPLAINING METHANOL}

A tentative conclusion from this discussion is that one can reasonably hope to learn about dust mantle composition by studying the abundance distribution of molecules in high temperature regions such as Orion-KL. This will not be easy! Some insight into the problems can be obtained from the article of Blake et al., 1987. They discuss in detail both the observations of this region and their interpretation. They conclude that while the nitrogen containing compounds seen in the hot core proper (i.e. $\mathrm{NH}_{3}, \mathrm{HC}_{3} \mathrm{~N}, \mathrm{C}_{2} \mathrm{H}_{5} \mathrm{CN}$, etc.) are mantle evaporation products, this is not the origin of methanol and other "compact ridge" molecules (i.e. dimethyl ether, methyl formate, etc.) which peak slightly to the south of the hot core.

As Figure 1 makes clear, the $\mathrm{CH}_{3} \mathrm{OH}$ abundance is one of the key characteristics of the chemistry of hot regions. In the Blake et al., picture, methanol is produced by mixing of ion-rich material from the region surrounding Orion-KL with water rich gas produced by shocks. Then radiative association of $\mathrm{CH}_{3}^{+}$and $\mathrm{H}_{2} \mathrm{O}$ gives rise to methanol. It is a little unclear what gives rise to this mixing but a test for the model might be to estimate ion abundances in the compact ridge source. Another test may be the relationship between deuterated species in the compact ridge (see Mauersberger et al., 1988). The ratio of $\mathrm{CH}_{2} \mathrm{DOH}$ to $\mathrm{CH}_{3} \mathrm{OD}$ could be informative since $\mathrm{H}_{2} \mathrm{O}$ (if produced by shocks) is presumably not $D$-enriched in the compact ridge but $\mathrm{CH}_{3}^{+}$may be. In any case (see Plambeck and Wright, 1988), the alternative presumably is to produce methanol also by evaporation which would be consistent with the indications that $\mathrm{CH}_{3} \mathrm{OH}$ is an abundant constituent of mantles (Tielens and Allamandola, 1987). This brings up the awkward question of whether $\mathrm{CO}$ can be converted to $\mathrm{CH}_{3} \mathrm{OH}$ on a grain surface. Both of these models then raise more questions than they answer.

\section{REFERENCES}

Beichman, C. A., et al. 1986, Ap. J., 307, 337.

Bell, M. B et al. 1988, Ap. J., \$26, 924.

Blake, G. A., Sutton, E. C., Masson, C. R., Phillips, T. G. 1987, Ap. J., \$15, 621.

Brown, P. D., Charnley, S. B., Millar, T. J. 1988, M. N. R. A. S., 231 ,409. 
Brown, R. D., Rice, E. H. N. 1986, M. N. R. A. S., 223 , 429.

d'Hendecourt, L. B., Allamandola, L., Greenberg, J. M. 1985, Astr. Ap., $152,136$.

Duley, W. W., Williams, D. A. 1984, Interstellar Chemistry, (London: Academic Press).

Goldsmith, P. F., Irvine, W., M., Hjalmarson, A., Ellder, J. 1986, Ap. J., $310,383$.

Henkel, C. et al. 1987, Astr. Ap., 182, 299.

Herbst, E. 1982, Astr. Ap., 111, 767.

Herbst, E., Leung, C. $1988, A p$. J. Suppl., in press.

Irvine, W. M., Goldsmith, P. F., Hjalmarson, A. 1987 in Interstellar Processes, eds. Hollenbach, D. J., Thronson, H. A. (Dordrecht: Reidel), p 561.

Jacq, T., Jewell, P. R., Henkel, C., Walmsley, C. M., Baudry, A. 1988, Astr. Ap., 199, L5.

Langer, W. D., Graedel, T. 1988, Ap. J. Suppl., in press.

Lepp, S., Dalgarno, A. 1984, Ap. J., 287, L47.

Mauersberger, R., Henkel, C., Jacq, T., Walmsley, C. M. 1988, Astr. Ap., 194, L1.

Millar, T. J., Bennett, A., Herbst, E. 1988, Ap. J., in press.

Millar, T. J. 1988, in Molecular Astrophysics, ed. Hartquist T. W., in press.

Moore, E. L., Langer, W. D., Huguenin, G. R. 1986, Ap. J., 306, 682.

Mundy, L. G., Scoville, N. Z., Baath, L. B., Masson, C. R., Woody, D. P. 1986, Ap. J., 304, L51.

Myers, P. C.. 1983, Ap. J., $270,105$.

Olano, C. A., Walmsley, C. M., Wilson, T. L. 1988, Astr. Ap., 196, 194.

Plambeck, R. L., Wright, M. H. C. 1987, Ap. J., s17, L101.

. 1988, Ap. J., 390, L61.

Tielens, A. G. G. M, Hagen, W. 1982, Astr. Ap., 114, 245.

Tielens, A. G. G. M 1983, Astr. Ap., 119, 177.

Tielens, A. G. G. M, Allamandola, L. J. 1987, in Physical Processes in Interstellar Clouds, NATO ASI Series, Vol 210, eds. Morfill, G. and Scholer, M., (Dordrecht: Reidel).

Walmsley, C. M., Wilson, T. L. 1984, in Nearby Molecular Clouds, ed. G. Serra, Lecture Notes in Physics 237, p 41.

Walmsley, C. M. 1985 in ESO-IRAM-ONSALA Workshop on (Sub) Millimeter Astronomy, ESO Conference and Workshop Proceedings, no 22, eds. Shaver P. A. and Kjar, K., p 327.

Walmsley, C. M. 1986, in Proceedings of the Enrico Fermi School on Interstellar Dust and Related Topics, Varenna, July 1986, in press.

Walmsley, C. M. et al. 1987, Astr. Ap., 172, 311.

Watson, W. D. 1976, Rev. Mod. Phys., 48, 513. 1978, Ann. Rev. Astr. Ap., 16, 585.

Welch, W. J. 1988, Astroph. Lett. and Comm., 26, 181.

Wilson, T. L., Johnston, K. J., Henkel, C., Menten, K. M. 1988, Astr. Ap., in press.

Wootten, A. 1986, in IAU Symposium 120: Interstellar Chemistry, eds. Tarafdar, S. and Vardya, M., (Dordrecht: Reidel). 


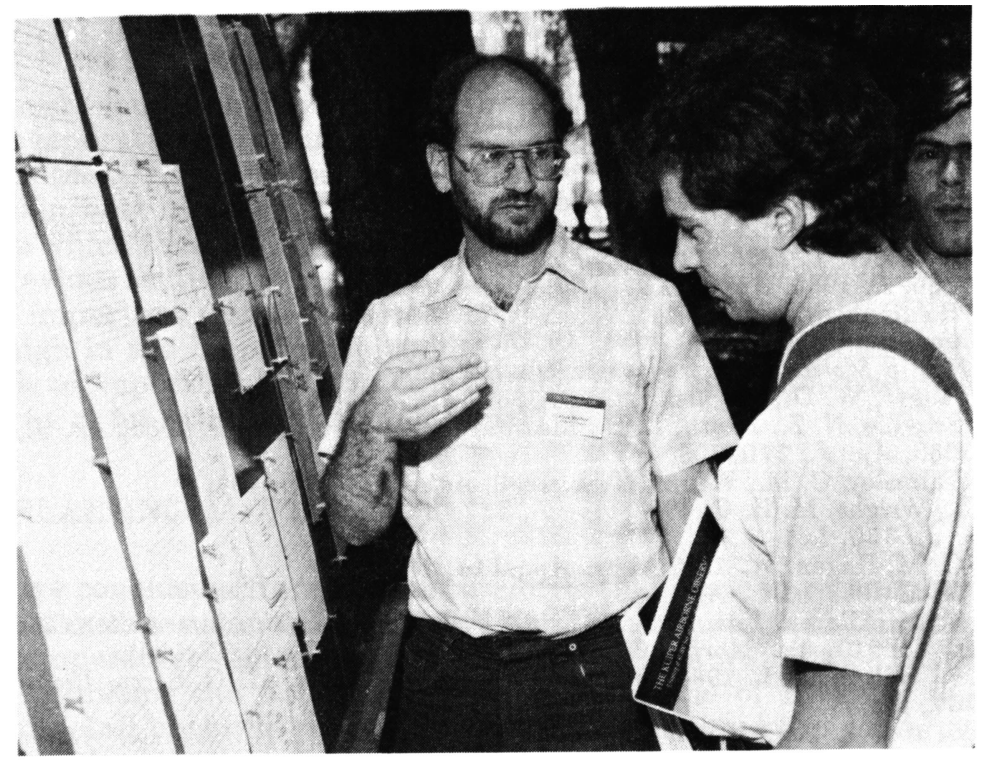

Mark Wolfire and Kurt Liffman 